\title{
Diffusion variétale du palmier à huile (Elaeis guineensis Jacq.)
}

\section{Dissemination of oil palm (Elaeis guineensis Jacq.) varieties}

Oléagineux, Corps Gras, Lipides. Volume 7, Numéro 2, 207-14, Mars - Avril 2000, Dossier : Afrique, plantation et développement

Auteur(s) : Tristan DURAND-GASSELIN, R. KOUAME KOUAME, B. COCHARD, B. ADON, P. AMBLARD

Résumé : La productivité du palmier à huile au cours du siècle passé a connu une croissance importante où l'amélioration génétique a pris une large part. Elle a connu un rythme de croissance assez comparable à celui obtenu pour des plantes annuelles de pays tempérés (blé, maïs, tournesol, etc.). Il est possible de dégager les étapes importantes qui ont marqué l'histoire de l'amélioration. Après une assez longue période de sélection massale, l'exploitation du gène " shell ", dont l'hérédité a été découverte par Beirnaert en 1939, a apporté une amélioration de 30 \% [1]. Vers 1960, l'exploitation de l'hétérosis des croisements inter-origines apporte une nouvelle amélioration de 10 $\%$ environ [2]. Enfin, deux cycles de sélection récurrente ont été achevés par différentes équipes et chacun a apporté 12 à $18 \%$ d'amélioration de la productivité. En 1991, la valeur moyenne des semences commerciales, plantées dans de bonnes conditions, était proche de 6,7 tonnes d'huile par hectare et par an [3]. Aujourd'hui, elle est de l'ordre de 7,2 t/ha/an [4]. Ces gains quantitatifs ont été accompagnés de progrès qualitatifs importants comme la diminution de la vitesse de croissance, l'augmentation du pourcentage d'huile dans les régimes ou l'acquisition d'une forte résistance à la fusariose en Afrique de l'Ouest [5]. La qualité du futur matériel végétal devrait être sensiblement améliorée par l'utilisation d'une base génétique élargie dans les programmes d'amélioration [6]. En effet, les stations de recherche disposent d'une bonne diversité génétique rassemblée lors de nombreuses prospections réalisées en Afrique (pour E. guineensis) et sur une espèce apparentée en Amérique latine (pour E. oleifera). Ces collections apportent de nouveaux caractères de qualité de l'huile, de résistance à la fusariose, qui sévit en Afrique, ou à la pourriture du cœur en Amérique latine. Le progrès génétique disponible en expérimentation doit être intégré dans les semences vulgarisées au plus vite et en quantité suffisante. Cependant, pour la reproduction du meilleur matériel, il existe des contraintes liées à la biologie des semences qui compliquent et ralentissent cette vulgarisation. En raison d'une dormance difficile à lever, les semences de palmier à huile sont distribuées sous forme de graines germées et leur distribution demande une organisation particulière.

Summary: Genetic improvement of the oil palm has been through several major stages. Mass selection was carried out before World War I. Around 1955, heterosis was found in crosses between selections carried out in Asia and those carried out in Africa. A reciprocal recurrent selection scheme was adopted in order to exploit it, and two selection cycles have been implemented since 1960. Varietal creation programmes make it possible to integrate the genetic progress made in trials and it is totally integrated into the seeds made commercially available. It has been estimated at a little over $40 \%$ since 1960. Over the same period, material resistant to Fusarium wilt has been selected. In Africa, where that disease is endemic, such material offers greater security for the investments made 
by oil palm growers. Given the dormancy in oil palm, which is difficult to break, oil palm seeds are supplied in germinated form. Their distribution requires special organization, especially for smallholders. The supply of improved planting material goes hand in hand with the development of oil palm plantations. It takes into account productivity requirements, hence grower income, and also attempts to secure investment by proposing appropriate material.

Keywords: palm tree, Elaeis guineensis, seeds, genetic improvement, recurrent selection, varietal creation programmes.

\section{ARTICLE}

\section{Historique de l'amélioration génétique}

II ne s'agit pas de reprendre en détail I'histoire de l'amélioration génétique du palmier à huile $[7,8]$ mais de donner les points essentiels qui expliquent la genèse des principales étapes de la sélection. Pour chaque époque, on aura ainsi une idée de la qualité de l'offre en matériel végétal amélioré.

\section{Sélections massales}

Dans les années 20, des sélections massales étaient effectuées en Asie du Sud-Est par les grandes plantations, au Congo Belge par I'INEAC et en Afrique occidentale française. Ces sélections ont donné, après deux à cinq générations, ce que Rosenquist appelle des " breeding populations of restricted origin " (BPRO) $)^{1}$. En Asie, ces sélections ont été assez efficaces et ont permis l'installation de grandes plantations (relativement pour l'époque). En Afrique, elles ont été perturbées par la disjonction d'un caractère contrôlant l'épaisseur de la coque (encadré 1). Les sélectionneurs réalisaient des croisements tenera par tenera qui étaient malheureusement fort peu intéressants car il y avait $25 \%$ de palmiers à fruits pisifera abortifs et $25 \%$ de dura. En Asie, où seuls des palmiers dura avaient été introduits en 1848 [8], ce problème ne se posait pas.

La compréhension de l'hérédité de ce caractère marque la fin de cette époque [1]. C'est aussi à cette époque que sont développées les premières techniques efficaces de fécondations contrôlées. La forte dépression de consanguinité dans les autofécondations sera bientôt évoquée (fin des années 40).

Expérience internationale (après-guerre 1946, plantée de 1950 à 1953)

Sur l'initiative de l'IRHO', cinq plantations échangent et intercroisent leurs meilleurs géniteurs, issus de leurs propres sélections, dans le cadre de ce que l'on a appelé l'« Expérience internationale ». Elle a donné des résultats tout à fait intéressants [2] : 


\section{Hérédité de l'épaisseur de la coque du fruit de palmier à huile}

L'épaisseur de a coque est contrôlée par un gène codominant $(\mathrm{Sh})$. Les arbres pisifera, homozygote sh- (absence de coque), ont le fruit le plus riche en pulpe et donc en huile, mais présentent une très forte stérilité femelle. Les arbres à type de fruit dura, homozygote $\mathrm{sh}^{+}$(coque épaisse), produisent $25 \%$ d'huile en moins que les hétérozygotes tenera (coque mince). Ces derniers sont donc les plus intéressants en plantation.

- mise en évidence d'une variabilité importante entre les différentes populations étudiées ;

- le caractère additif de l'hérédité du nombre et du poids moyen des régimes produits par les palmiers, composantes essentielles de la production ;

- la supériorité des croisements inter-origines sur les croisements intra-origines en particulier lorsqu'il y a complémentarité (typiquement l'origine Deli ${ }^{3}$, développée en Asie, qui produit un petit nombre de gros régimes se combine bien avec l'origine la Mé, développée en Côte d'Ivoire, qui produit un grand nombre de petits régimes). Ce résultat a été exploité pour la production de semences [9].

\section{Premier cycle de sélection}

Pour exploiter l'hétérosis existant dans les croisements réalisés entre certaines origines et les contraintes dues à la dépression de consanguinité, I'IRHO adopte dès 1957 un schéma de sélection récurrente réciproque inspiré des travaux réalisés sur le maïs [10]. Le matériel est divisé en deux groupes $\mathrm{A}$ et $\mathrm{B}$ selon la complémentarité des caractères et son aptitude à bien se combiner avec le groupe réciproque [11].

Schématiquement, le groupe $A$ inclut les origines à petit nombre de gros régimes - le matériel initialement développé en Asie : Deli Socfin, Deli Guthrie, Deli Dabou et quelques populations africaines : Angola... - et le groupe $B$ les origines à grand nombre de petits régimes - origines d'Afrique centrale : Yangambi (RDC ex-Zaïre), Sibiti (Congo) ou de l'Ouest : la Mé (Côte d'Ivoire), Pobè (Bénin), Nigeria...

De 1959 à 1970, 529 croisements A x B sont plantés à la Mé (CNRA) ${ }^{4}$ et au Cameroun. Ces croisements exploitent une bonne partie du matériel réuni lors de l'Expérience internationale et quelques introductions ultérieures. Il est réalisé sur une base génétique relativement large.

Les principaux résultats ont été :

- le calcul de l'héritabilité des caractères dont une synthèse a été faite par Meunier [12] ; 
- l'exploitation du premier cycle pour la sortie variétale a retenu quinze croisements [13].

Le progrès génétique réalisé au cours de ce cycle de sélection a été entièrement valorisé dans la sortie variétale à partir de 1976 [14]. Alors que la moyenne des croisements issus de l'expérience internationale produisait environ 2 tonnes d'huile par hectare, la moyenne des croisements du premier cycle produisait 3,3 tonnes d'huile par hectare et ceux sélectionnés après application d'une régression géno-phénotypique 3,6 tonnes d'huile par hectare. Les conditions climatiques se sont dégradées depuis (augmentation du déficit hydrique) et ces valeurs seraient aujourd'hui respectivement de 3 et 3,3 tonnes d'huile par hectare (tableau 1).

Dans le cycle de sélection suivant, on a cherché à renforcer cet acquis important.

\section{Second cycle de sélection}

Une grande partie du second cycle de sélection avait pour but d'exploiter la variation intrafamiliale des autofécondations (encadré 2). Un cycle de sélection généalogique est réalisé à partir d'un croisement $A \times B$. Typiquement on compare des croisements réalisés entre les arbres autofécondés de $A$ et les arbres autofécondés de $B$. On est assuré d'un progrès génétique important tout en restant proche d'un matériel dont le comportement agronomique est bien connu. Dans la mesure où on dispose des autofécondations pour la sortie variétale, ce cycle de sélection peut être mis en place avant un cycle normal avec recombinaisons [14].

Par extension et puisque la sortie variétale l'utilisait [13], des essais ont été plantés pour améliorer des ensembles du type $\left(A_{1} \times A_{2}\right) \times B_{A F}$ ou encore $A_{A F} \times\left(B_{1} \times B_{2}\right)$. Plus rarement, on a réalisé des essais $\left(A_{1} \times A_{2}\right) \times\left(B_{1} \times B_{2}\right)$. Ces quelques recombinaisons s'appuyaient cependant sur une base génétique assez étroite. De plus, elles étaient presque toujours intra-« BPRO ». Au total à fin 1992, le second cycle comprenait plus de 1300 croisements (presque autant d'hectares) plantés dans un réseau d'essais répartis en Afrique, Asie et Amérique latine.

Pour être plus complet, il faudrait ajouter des essais à but particulier qui ont été plantés au même moment dans le but d'étudier certains paramètres génétiques [17].

\section{Résistance à la fusariose}

La fusariose est une maladie fongique provoquée par Fusarium oxysporum f.sp. elaeidis (Foe). Elle est endémique en Afrique et c'est un des principaux facteurs susceptibles de compromettre un investissement palmier. En première génération elle se déclare tardivement dans les plantations. En revanche, en replantation, les palmiers sont confrontés à un inoculum important et la fusariose s'exprime au jeune âge $[5,18,19]$. 


\section{En cadré 2}

\section{Bologie florale du palmier à huile}

Le palmier à huile est une plante monoïque temporelle. L'arbre émet alternativement des séries d'inflorescences mâles ou femelles: c'est une plante naturellement allogame. Les arbres utilisés dans les programmes d'amélioration sont très hétérozygotes et il subsiste dans kur autofécondation une variance génétique importante $[15,16]$.

La sélection de matériel résistant à la fusariose est actuellement la seule méthode de lutte satisfaisante. Pour cela, on dispose d'un test de résistance précoce dès la prépépinière, développé au début des années 70 [20]. Ces résistances partielles, de degrés divers, se rencontrent dans presque toutes les populations de palmier à huile [21]. En Côte d'Ivoire, on rencontre des souches de Fusarium particulièrement virulentes qui rendent très efficaces les sélections; de plus, l'absence d'interactions entre les isolats de zones géographiques différentes et les génotypes de palmier à huile [22] autorise la diffusion au niveau régional de matériel résistant.

Ainsi, dans les conditions de Côte d'Ivoire, la fusariose n'est désormais plus un problème majeur, mais elle le reste potentiellement. En replantation, il est recommandé de ne planter que du matériel résistant dès que des cas de fusariose ont été observés à la génération précédente et de toujours éviter le matériel sensible.

\section{Conclusion partielle}

Le tableau 1 résume l'historique des principales étapes de la sélection et donne une idée de la progression de la productivité du matériel végétal vulgarisé de même que la figure 1 . Cette progression, en 40 ans, a été un peu supérieure à $40 \%$, soit environ $1 \%$ par an même si, pour diverses raisons, l'augmentation de la productivité ces dernières années a été un peu moins forte.

Outre la production en huile d'autres facteurs ont été pris en compte :

- croissance en hauteur faible qui facilite la récolte ;

- résistance à la fusariose pour l'Afrique, qui doit replanter de vastes superficies dans les prochaines années [23] ;

- taux d'extraction (teneur en huile des régimes) qui augmente l'efficacité de la première transformation et permet une meilleure valorisation de la production.

Ce progrès génétique a sans doute participé, en assurant un revenu plus important aux planteurs, à l'essor de la culture du palmier à huile en Afrique (voir l'article de Naï Naï, Cheyns et Ruf).

Les progrès futurs proviendront de l'exploitation d'une base génétique élargie. 


\section{Production de semences : programme et réalisation}

Élaboration d'un programme de production de semences

\section{Choix des catégories hybrides ${ }^{5}$}

Tester un croisement dans de bonnes conditions demande à peine moins d'un hectare. Un cycle de sélection demande 1000 à 2000 hectares au moins qui seront immobilisés pour 25 ans. Il est donc presque nécessaire de travailler en réseau. Tester des croisements dans des écologies différentes peut entraîner des difficultés de comparaison. Pour le matériel sur lequel nous avons travaillé, il a été vérifié l'absence d'interaction génotype-environnement significative [24]. II a été aussi vérifié que les résultats peuvent s'exprimer en pourcentage par rapport à un témoin. On regroupe les résultats par catégorie hybride, et leur valeur est évaluée sur un ensemble de caractères : production, croissance en hauteur, résistance aux maladies, encombrement, etc. (tableau 2).

\section{Choix des croisements}

La valeur exacte d'un croisement est estimée avec une certaine incertitude liée aux conditions expérimentales (nombre d'arbres testés, homogénéité du terrain, etc.). Pour cette raison on retient dans chaque catégorie hybride sélectionnée plusieurs croisements car leur valeur moyenne est connue avec plus de précision.

L'intensité de sélection (nombre de croisements retenus sur nombre de croisements observés) est variable au sein de chaque catégorie hybride. Il est également tenu compte des aptitudes à la combinaison des parents : un parent dont l'aptitude à la combinaison est simplement moyenne peut être avantageusement remplacé par un arbre ayant une bonne aptitude générale à la combinaison. Ainsi, les différences entre catégories restent minimes et ne sont jamais significatives.

L'exemple du choix des croisements dans deux catégories hybrides largement diffusée : " C1001 » (DA 115 D AF x LM 2 T AF) et « C2501 » [(DA 5 D x DA 3 D) x LM 2 T AF] est donné dans le tableau 3.

\section{Réalisation du programme}

\section{Reproduction d'un croisement}

Refaire un croisement $A \times B$ ne permet pas d'obtenir un nombre suffisant de semences. Mais, un effet multiplicateur important est obtenu en croisant les deux ensembles d'arbres issus de l'autofécondation de chacun des parents.

Pour obtenir $100 \%$ d'arbres de type tenera, des arbres dura issus de l'autofécondation du parent dura, sont croisés par des arbres pisifera issus de l'autofécondation du parent tenera (figure 2). Ces derniers sont nécessairement utilisés comme parent mâle puisqu'ils sont femelles stériles. Pour chaque croisement, on utilise quelques dizaines de dura (50 à 200) qui sont croisés à un échantillon minimum de 10 à 30 pisifera.

Si le croisement testé est un dura par pisifera, on utilise comme précédemment les arbres issus de l'autofécondation du dura mais ils sont croisés directement avec le pollen du parent pisifera qui, lui, 
ne peut pas être autofécondé. Parfois le nombre de semences produites de cette façon est limité par la disponibilité du pollen mais ce problème a été en grande partie résolu [4].

\section{Délais de diffusion du progrès génétique}

Le palmier à huile est une plante pérenne et la gestion du temps est un facteur qui requiert une attention particulière. Pour réduire le décalage observé entre les résultats de la création variétale et leur prise en compte dans les programmes de fourniture de semences, il faut anticiper sur les résultats. Pour cela, les autofécondations dura et tenera sont plantées dès les premières années des tests d'évaluation (tableau 4). En théorie, les résultats d'un essai sont donc pleinement intégrés dans la production de semences 4 ans après sa conclusion. Mais comme un cycle de sélection comprend plusieurs blocs génétiques et que chacun est planté en plusieurs années (5 à 6), on comprend qu'il existe un décalage de 10 ans environ entre la valeur du matériel en station et la valeur des semences commerciales [14].

\section{Contrôles de la qualité}

Comme toute production de semences, celle du palmier à huile demande une organisation rigoureuse et des procédures de contrôle efficaces. Elles ont été mises en place dès le début des années 60 et ont subi quelques améliorations depuis. Les principales portent sur :

- l'isolement des fleurs : qualité des sacs d'isolement (tissu), précaution de temps (isoler un temps suffisant avant et après la date d'anthèse) ;

- la qualité du pollen utilisé, le pouvoir germinatif et la durée de conservation limitée ;

- la qualité de l'étiquetage et la conservation des informations ;

- le contrôle de la qualité des isolements et de l'ensemble du travail par la réalisation de fécondation à blanc : lorsque toutes les précautions ont été respectées, il n'y a aucun fruit noué...

\section{Distribution des semences}

\section{Germination des semences}

Les graines de palmier sont semi-récalcitrantes. Légèrement déshydratées (humidité de 8 à $10 \%$ du poids de la graine entière), elles peuvent être conservées 2 ans environ mais perdent lentement, à partir du $12^{\mathrm{e}}$ mois, leur capacité à germer. Leur pseudo-dormance peut être levée par un traitement à la chaleur sèche [25] dont les principales étapes sont :

- un trempage suivit d'un ressuyage pour amener les semences à $18 \%$ d'humidité sur poids de la graine entière ;

- un chauffage à $39 \pm 1{ }^{\circ} \mathrm{C}$ des graines préalablement placées dans des sacs fermés hermétiquement. Le chauffage dure 80 jours ;

- un nouveau trempage et ressuyage pour amener les semences à $21 \%$ d'humidité sur poids de la graine entière.

La germination est alors obtenue en 2 à 4 semaines à température ambiante $\left(27^{\circ} \mathrm{C}\right.$ en moyenne sous 
les tropiques).

Ce traitement demande des installations particulières et ne peut être réalisé que par des intervenants spécialisés. Une fois la graine germée et différenciée, il est bon de la repiquer en terre au plus tôt. Certains gros planteurs peuvent recevoir les graines après le chauffage et assurer euxmêmes la phase de germination qui ne demande pas d'installation particulière.

\section{Livraison des semences aux planteurs : cas de la Côte d'Ivoire}

Le palmier sélectionné est distribué principalement sous forme de graines germées, produit périssable. Il faut donc faire coïncider au mieux l'offre et la demande.

Les sociétés agro-industrielles passent leurs commandes à l'avance et il est assez facile de bâtir avec eux un échéancier des livraisons.

Pour le secteur villageois, en Côte d'Ivoire, des sociétés d'État ont servi d'intermédiaires pendant de nombreuses années à la station de recherche de la Mé qui est seule équipée d'un germoir. Aujourd'hui, après la privatisation des grands ensembles agro-industriels, la station de la Mé assure directement la livraison aux petits planteurs, ce qui est bien plus difficile à gérer. II est difficile d'évaluer à l'avance la demande et de programmer les livraisons. Donner des rendez-vous aux planteurs n'a pas suffi puisque la plupart ne se présentent pas à la date convenue. Mais, à la longue, un certain équilibre s'instaure entre les planteurs " avec » et " sans » rendez-vous ; toutefois, il est illusoire de vouloir gérer la demande avec précision.

Les livraisons aux petits planteurs sont devenues très importantes dès 1994, et s'y sont ajoutés quelques programmes de développement gérés par l'état. La figure 3 donne une idée des surfaces nouvelles qui ont été créées en théorie (en convertissant le nombre de graines achetées en hectare). De fait, on retrouve bien ces surfaces ${ }^{6}$ dans les inventaires des plantations villageoises réalisés par les ensembles agro-industriels qui sont appelés à traiter les récoltes (voir l'article de Naï Naï, Cheyns et Ruf).

Entre 1998 et 1999 ce sont plus de 5000 planteurs qui ont été servis en semences sélectionnées.

À l'occasion de la livraison des semences aux planteurs, il est possible d'élaborer quelques statistiques simples qui seront utiles aux opérateurs de la filière : organisations professionnelles agricoles et agro-industries. Les fruits du palmier à huile, une fois récoltés, ne peuvent être stockés. II convient donc d'adapter les capacités d'usinage, petites ou grandes unités, aux productions attendues. L'évolution du verger est observée par région et par type de plantations : nouvelles créations, extension de plantations existantes et replantation d'une vieille palmeraie (tableau 5).

\section{Conseil aux planteurs}

II ne revient pas directement aux producteurs de semences de conseiller les planteurs. La vente de semences est cependant l'occasion de donner aux planteurs des informations auxquelles ils n'ont pas toujours accès. Elles portent généralement sur les points suivants :

- techniques de prépépinière et pépinière ; 
- qualité du matériel végétal et mise en garde sur la valeur médiocre du matériel « tout venant ${ }^{7}$;

- proposition de semences résistantes à la fusariose pour les planteurs situés dans des zones à risque ou qui effectuent une replantation d'une vieille palmeraie.

\section{Perspectives}

La production de matériel végétal sélectionné de plus en plus performant a accompagné tout au long de ce siècle le développement des plantations de palmier à huile. Si la culture de cette plante reste, au niveau mondial, encore très liée au développement de l'agro-industrie, la part des petits planteurs ne cesse de croître. L'offre en matériel végétal doit en tenir compte. Il faut sécuriser l'investissement des planteurs dans le cadre de leur environnement socio-économique.

Le palmier à huile reste encore une plante rustique qui n'a finalement subi que deux véritables cycles d'amélioration génétique contre 50 ou 100 réalisés pour des plantes annuelles. À condition de diffuser un nombre suffisant de catégories hybrides, chacune représentée par plusieurs croisements, on conserve au matériel végétal une bonne homéostasie.

Cependant, à moyen terme, la sortie variétale pourrait être fondée sur la reproduction d'individus et non plus de croisements. En effet, la variance résiduelle intra-familiale peut être exploitée en clonant, par multiplication végétative in vitro, les individus exceptionnels d'un croisement. Cela offre des potentialités remarquables pour l'amélioration de la productivité et des qualités du matériel végétal. Le risque serait de perdre en sécurité ce que l'on gagne en productivité ${ }^{8}$. Cependant, par exemple pour la résistance aux maladies, les clones peuvent apporter une sécurité renforcée. Ainsi deux points importants ont été mis en évidence lors des premiers tests de clones pour la résistance à la fusariose :

- quelques clones sont bien plus résistants que la moyenne des meilleurs croisements et semblent particulièrement indiqués pour replanter les zones où l'incidence de la fusariose était très forte en première génération ;

- il existe des clones résistants issus de croisement relativement sensibles : la base génétique du matériel résistant s'en trouve élargie.

Des programmes sont également en cours pour rechercher des résistances à la pourriture du cœur qui occasionne des dégâts extrêmement importants en Amérique latine [26], ou encore au ganoderma, maladie largement répandue en Asie [27] mais aussi en Afrique centrale. Certains clones pourraient y apporter des solutions originales.

Dans le futur, il faudra prendre en compte tous ces aspects et éviter de concentrer la sortie variétale sur une base génétique trop étroite. L'intégration d'outils nouveaux comme la sélection assistée par marqueur aidera les sélectionneurs à gérer la variabilité dans leur programme d'amélioration.

Notes

${ }^{1}$ Populations améliorées à base génétique étroite (Par exemple : Marihat, Dolok Sinumbah, Bah Lias en Indonésie ; Elmina, Chemara, Socfin en Malaisie ; Yangambi, Ekona, la Mé en Afrique et bien d'autres encore). 
${ }^{2}$ Institut de recherches pour les huiles et oléagineux.

${ }^{3}$ Deli est une région de Nord Sumatra (Indonésie).

${ }^{4}$ Centre national de recherche agronomique de Côte d'Ivoire.

${ }^{5}$ Sous ce terme, on regroupe l'ensemble des croisements ayant les mêmes grand-parents.

${ }^{6}$ Avec un décalage d'un an environ, le planteur devant réaliser une pépinière avant plantation.

${ }^{7}$ Des graines germées généralement ramassées dans les plantations commerciales sont souvent proposées aux planteurs à prix réduit. Le plus souvent il s'agit de croisements tenera par tenera dont la production attendue (en huile) est estimée entre 30 et $40 \%$ de celle des graines sélectionnées.

${ }^{8}$ Si le matériel végétal avait une base génétique étroite (par exemple un seul clone), il serait a priori plus sensible aux aléas climatiques et aux maladies.

\section{REFERENCES}

1. BEIRNAERT A, VANDERWEYEN R (1941). Contribution à l'étude génétique et biométrique des variétés d'Elaeis guineensis Jacquin. Publication de l'INEAC, série scientifique, $\mathrm{n}^{\circ} 27$.

2. GASCON JP, DE BERCHOUX C (1964). Caractéristiques de la production d'Elaeis guineensis (Jacq.) de diverses origines et de leurs croisements : application à la sélection du palmier à huile. Oléagineux, $19:$ 75-84.

3. NOUY B, LUBIS RA, et al. (1991). Potentiel de production chez le palmier à huile Elaeis guineensis. Oléagineux, 46 : 91-9.

4. DURAND-GASSELIN T, NOIRET JM, et al. (1999). Disponibilité de pollen performant pour la production de semences améliorée de palmier à huile (Elaeis guineensis Jacq.). Plantations, Recherche, Développement, $6: 264-76$.

5. DE FRANQUEVILLE H, RENARD JL (1990). Bilan de l'amélioration du niveau de tolérance du palmier à huile à la fusariose. Évolution de la maladie sur la plantation R. Michaux. Oléagineux, 45 : 399-405.

6. DURAND-GASSELIN T, BAUDOUIN L, et al. (1999). Effets de l'autofécondation et de la recombinaison sur l'expression de la variabilité génétique chez le palmier à huile. Conséquence pour I'amélioration génétique. Plantations, Recherche, Développement, 6 (in press).

7. HARTLEY CWS (1988). The oil palm (Elaeis guineensis Jacq.). Essex, Longman Scientific \& Technical.

8. ROSENQUIST EA (1985). The genetic base of oil palm breeding populations. Works shop on oil palm germplasm and utilisation. Selengor, Malaysia, Palm oil research institute of Malaysia.

9. BÉNARD G, MALINGRAUX C (1965). La production de semences sélectionnées de palmier à huile à I'IRHO. Principe et réalisation. Oléagineux, 20 : 297-302. 
10. COMSTOCK RE, ROBINSON HF, et al. (1949). A breeding procedure designed to make maximun use of both general and specific combining ability. Agron J, $41: 360$.

11. MEUNIER J, GASCON JP (1972). Le schéma général d'amélioration du palmier à huile à l'IRHO. Oléagineux, 27 : 1-12.

12. MEUNIER J, GASCON JP, et al. (1970). Hérédité des caractéristiques du régime d'Elaeis guineensis Jacq. en Côte d'Ivoire - hérédité. Aptitude à la combinaison. Oléagineux, 25 : 377-82.

13. GASCON JP, JACQUEMARD JC, et al. (1981). La production de semences séléctionnées de palmier à huile Elaeis guineensis. Oléagineux, 36 : 475-86.

14. Gascon JP, Le Guen V, et al. (1988). Résultats d'essais de second cycle de sélection récurrente réciproque chez le palmier à huile Elaeis guineensis Jacq. Oléagineux, 43.

15. BEIRNAERT A (1935). Introduction à la biologie florale du palmier à huile (Elaeis guineensis Jacquin). Publication de l'Institut national pour l'étude agronomique du Congo belge. INEAC 5 : 1-42.

16. HENRY P (1957). Recherches sur la croissance et le développement chez Elaeis guineensis Jacq. et chez Cocos nucifera L., comparaison avec quelques autres palmiers. Faculté des sciences. Paris, Thèse, Université de Paris ; $154 \mathrm{p}$.

17. CAO TTV (1995). Organisation de la variabilité génétique chez le palmier à huile (Elaeis guineensis Jacq.). Conséquences pour l'amélioration des populations et la création variétale. Institut national agronomique. Paris Grignon, Institut national agronomique : 200.

18. RENARD JL, DE FRANQUEVILLE H, et al. (1993). Étude de l'incidence de la fusariose vasculaire sur la production de régimes, chez le palmier à huile. Oléagineux, 48 : 495-504.

19. DE FRANQUEVILLE H, DIABATÉ S (1995). La fusariose du palmier à huile en Afrique de l'Ouest. Plantations, Recherche, Développement, 2 : 5-10.

20. RENARD JL, GASCON JP, et al. (1972). Recherches sur la fusariose du palmier à huile. Oléagineux, 27: 581.

21. DURAND-GASSELIN T, DE FRANQUEVILLE $H$, et al. (2000). Assessing and utilising sources of resistance to Fusarium wilt in oil palm (Elaeis guineensis Jacq.) genetic resources. Proceeding of the " International conference on science and technology for managing plant genetic diversity in the 21 st Century » 12-16 June 2000, Kuala Lumpur (Malaysia).

22. FLOOD J, MEPSTED R, et al. (1993). Comparison of virulence of isolates of Fusarium oxysporum f.sp. elaeidis from Africa and South America. Plant Pathol, 42 : 168-71.

23. HIRSCH R. (1996). Les filières palmier à huile et hévéa dans la zone franc. Enquête sur la relance des économies de la zone franc après la dévaluation. Paris, France, ministère de la Coopération, CFD.

24. COCHARD B, NOIRET JM, et al. (1993). Second cycle reciprocal recurrent selection in oil palm, Elaeis guineensis Jacq. Results of deli x La Mé hybrids tests. Oléagineux, 48 : 441-51. 
25. CORRADO F, WUIDARD W (1990). Germination des graines de palmier à huile (E. guineensis) en sacs de polyéthylène. Méthode par « chaleur sèche ». Oléagineux, 45 : 511-5.

26. MARIAU D, VAN DE LANDE HL, RENARD JL, et al. (1992). Les maladies de type pourriture du cœur sur le palmier à huile en Amérique latine : symptomatologie, épidémiologie, incidence. Oléagineux, $47: 605-98$.

27. WOOD BJ (1983). Oil palm pests. Exotic plant quarantine pests \& procedures for introduction of plant materials. KG Singh Serdang, Selangor, Malaysia, Asean plant quarantine centre and training institute : 53-68.

\section{Illustrations}

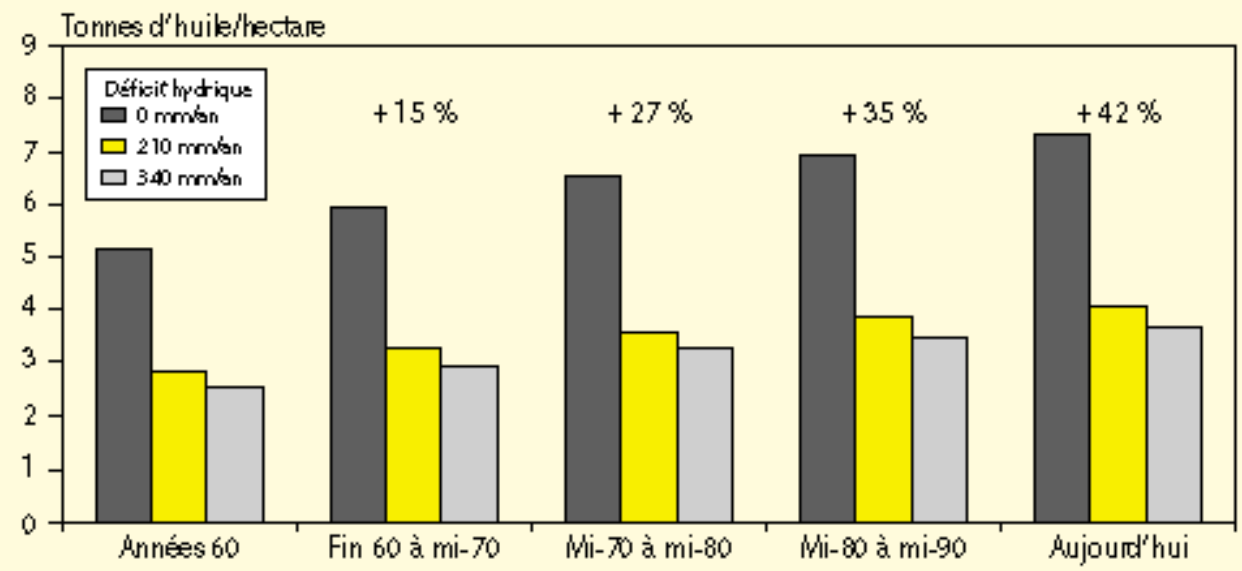

Figure 1. Évolution du potentiel de production des semences de palmier à huile vulgarisées. 
Reproduction d'un croisement sélectionné $\mathrm{A} \times \mathrm{B}$.

(A est dura, B est tenera)

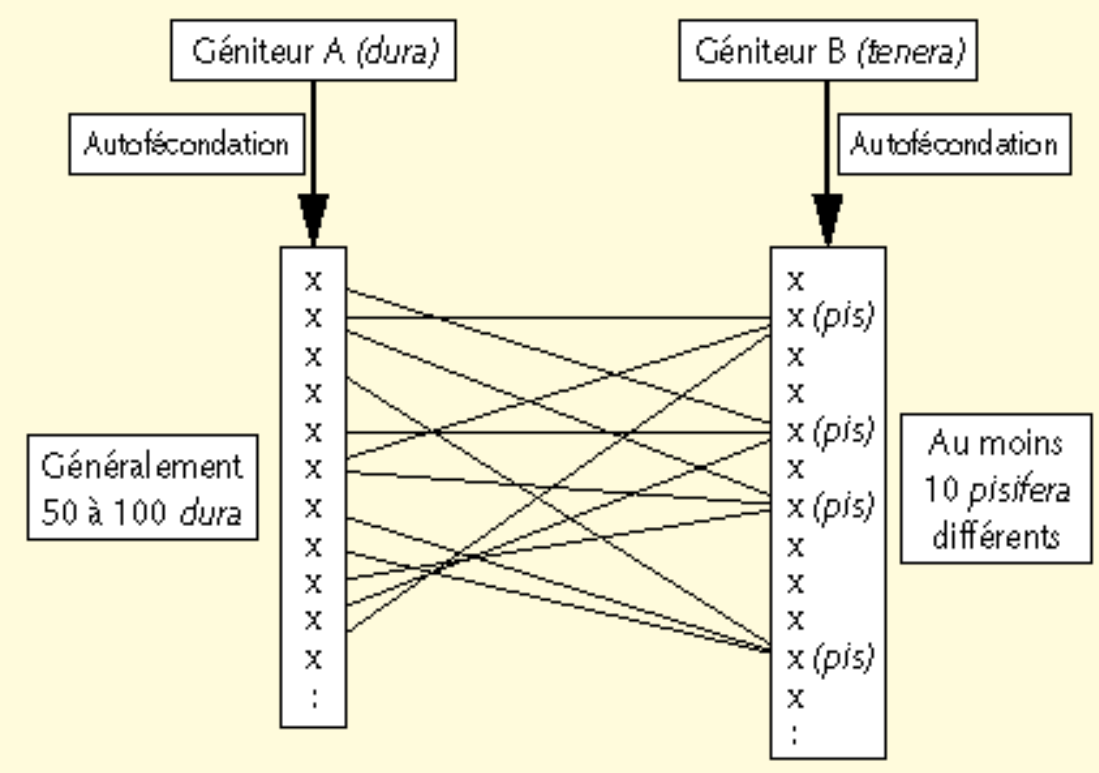

Figure 2. Représentation schématique de la reproduction d'un croisement pour la production de semences.

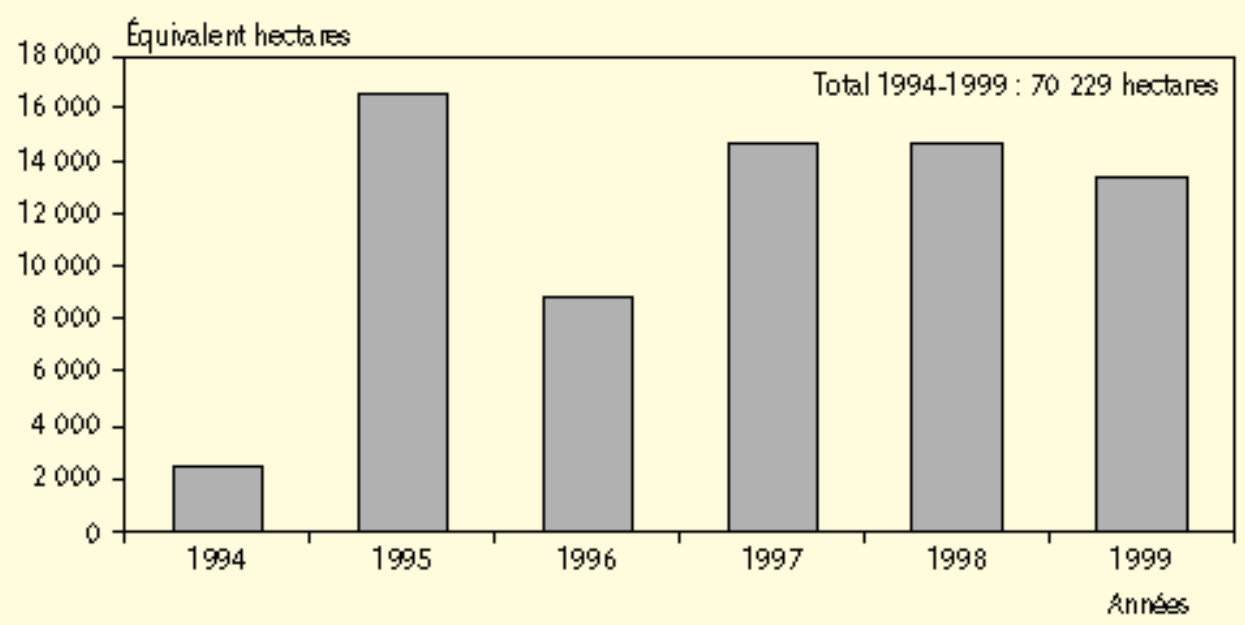

Figure 3. Livraison de semences sélectionnées aux petits planteurs en Côte d'lvoire : il faut théoriquement 200 graines germées pour créer un hectare de plantation (143 palmiers). 


\begin{tabular}{|c|c|c|c|c|}
\hline \multirow{2}{*}{$\begin{array}{l}\text { Cycles } \\
\text { (Années de plantation) }\end{array}$} & \multirow{2}{*}{$\begin{array}{l}\text { Valeur en station } \\
\text { b (en t/ha d'huile) } \\
\text { (résultats } 9 \text { ans } \\
\text { après plantation) }\end{array}$} & \multicolumn{2}{|c|}{$\begin{array}{l}\text { Valeur de la sortie variétale } \\
\text { b (en t/ha d'huile) }\end{array}$} & \multirow[t]{2}{*}{ Diffusion } \\
\hline & & $\begin{array}{c}\text { Condition s clim atiques } \\
\qquad \text { avant } 1970^{\mathrm{a}}\end{array}$ & $\begin{array}{c}\text { Condition s clim atiques } \\
\text { actuelle }\end{array}$ & \\
\hline \multicolumn{5}{|l|}{ Expérience } \\
\hline internationa le (1950-1953) & 2 & $\begin{array}{l}\text { Croisements } \\
\text { inter-origines : } 2,9\end{array}$ & $\begin{array}{l}\text { Croisements } \\
\text { inter-origines : } 2,6\end{array}$ & $\begin{array}{l}\text { Utilisé jusque } \\
\text { vers } 1968^{c}\end{array}$ \\
\hline \multirow[t]{2}{*}{ Premier cycle de sélection (1959-1968) } & 3,3 & Premiers résultats : 3,3 & Premier résultats : 3,0 & $\begin{array}{l}\text { Diffusé à partir } \\
\text { de la fin } \\
\text { des années } 60\end{array}$ \\
\hline & & Demiers résultats : 3,6 & Demiers résultats : 3,3 & $\begin{array}{l}\text { Diffusé à partir } \\
\text { de } 1976\end{array}$ \\
\hline \multirow[t]{2}{*}{ Second cycle de sélection (1975-1986) } & 3,6 & Premiers résultats : 3,9 & Premiers résultats : 3,5 & $\begin{array}{l}\text { Diffusé à partir } \\
\text { de 1984-1985 }\end{array}$ \\
\hline & & Nlle sélection : 4,1 & Nlle sélection : 3,7 & $\begin{array}{l}\text { Diffusé à partir } \\
\text { de } 1998\end{array}$ \\
\hline
\end{tabular}

Tableau 1. Historique du matériel végétal disponible en Côte d'lvoire pour les planteurs et indication de sa valeur relative (en pratique, on passe progressivement d'une étape à l'autre)

a Déficit hydrique de $210 \mathrm{~mm} / \mathrm{an}$ en moyenne.

${ }^{\mathrm{b}}$ Déficit hydrique de $340 \mathrm{~mm} / \mathrm{an}$ en moyenne.

c Quelques croisements dura x tenera étaient encore diffusés à la demande des clients. 


\begin{tabular}{|c|c|c|c|c|c|}
\hline \multicolumn{6}{|c|}{ Catégorie hybrides } \\
\hline $\begin{array}{c}\text { Gran ds-parents } \\
\text { Groupe A } \\
\text { (Autofécondation ou croisement) }\end{array}$ & $\begin{array}{l}\text { Grands-parents } \\
\text { Groupe B } \\
\text { (Autofécondation ou croisement) }\end{array}$ & $\begin{array}{l}\text { Nombre } \\
\text { croist }^{t}\end{array}$ & $\begin{array}{c}\text { Huile } \\
\text { en \% tém oin }\end{array}$ & $\begin{array}{l}\text { Production } \\
\text { de régimes } \\
\text { en \% tém oin }\end{array}$ & $\begin{array}{l}\text { Huile sur } \\
\text { régimes en } \\
\text { \% tém oin }\end{array}$ \\
\hline \multicolumn{6}{|l|}{ Gatégories retenues } \\
\hline DA 3D AF & LM2T AF & 22 & 117 & 101 & 116 \\
\hline DA5DAF & LM 5 T AF & 11 & 116 & 101 & 115 \\
\hline DA 3D AF & LM2 T x LM10 T/LM 5T & 16 & 113 & 98 & 116 \\
\hline DA $115 \mathrm{D} \times \mathrm{DA} 3 \mathrm{D}$ & LM2 T AF/LM10 TAF & 16 & 113 & 97 & 117 \\
\hline LM 404 D x DA 115 D & LM 2 T x LM 10 T/LM 5T & 8 & 113 & 99 & 114 \\
\hline LM 404 D x DA 10 D & LM10 T AF & 11 & 113 & 100 & 113 \\
\hline DA 5D x DA 3 D & LM2 T AF & 40 & 113 & 105 & 107 \\
\hline DA 5D $\times$ DA 3 D & LM 5 T x LM 10 T & 7 & 112 & 97 & 116 \\
\hline DA115 D AF & LM2T AF & 78 & 112 & 100 & 111 \\
\hline LM 404 D AF & LM $2 \mathrm{~T} \mathrm{AF}$ & 67 & 110 & 94 & 117 \\
\hline LM404 Dx DA115 D & LM10 T AF & 11 & 109 & 97 & 112 \\
\hline \multicolumn{6}{|c|}{ Gatégories retenues maisoù Yintensité de sélection sera forte } \\
\hline LM407 D AF & LM451 TAF & 26 & 108 & 96 & 111 \\
\hline LM 404 D x DA 10 D & LM 2 T AF & 66 & 107 & 100 & 108 \\
\hline DA118 D AF & LM2T AF & 16 & 107 & 95 & 112 \\
\hline $\mathrm{DA} 10 \mathrm{D} \times \mathrm{DA} 3 \mathrm{D}$ & LM 2 T AF & 34 & 107 & 101 & 105 \\
\hline DA 8 D $\times$ LM $269 \mathrm{D}$ & LM2T AF & 18 & 106 & 103 & 103 \\
\hline DA5DAF & LM $2 \mathrm{~T}$ x SI 10T & 21 & 106 & 96 & 110 \\
\hline LM 269 Dx DA 115 D & LM 2 T AF & 34 & 103 & 95 & 108 \\
\hline \multicolumn{6}{|l|}{ Catégories éliminées } \\
\hline LM 404 D AF & LM718 TAF & 9 & 102 & 85 & 120 \\
\hline DA118 D AF & LM451 TAF & 32 & 101 & 89 & 113 \\
\hline DA10 D AF & LM 2 T AF & 165 & 101 & 101 & 100 \\
\hline LM 269 D AF & LM 2 T AF & 35 & 100 & 92 & 108 \\
\hline DA $118 \mathrm{D} \times \mathrm{DA} 300 \mathrm{D}$ & LM451 TAF & 33 & 99 & 93 & 107 \\
\hline PO 498 D AF & LM451 TAF & 34 & 90 & 88 & 103 \\
\hline DA 22D $\times$ DA 3D & LM451 TAF & 10 & 79 & 79 & 101 \\
\hline
\end{tabular}

Tableau 2. Caractéristiques de production des principales catégories hybrides du second cycle de sélection 


\begin{tabular}{|c|c|c|c|}
\hline Dura & Tenera ou pisifera & $\begin{array}{l}\text { Valeur production } \\
\text { d'huile (\% témoin) }\end{array}$ & $\begin{array}{l}\text { Taux d'extraction } \\
\text { (\% témoin) }\end{array}$ \\
\hline \multicolumn{4}{|c|}{ Catégorie hybride C 1001} \\
\hline LM 2514 D & LM1 578P, LM2275P & 113 & 116 \\
\hline LM 2347 D & LM2286T & 116 & 113 \\
\hline LM 2526 D & LM1 595P & 117 & 114 \\
\hline LM 2536 D & LM1 578P, LM2448T & 116 & 113 \\
\hline LM 2517 D & LMI 600P & 117 & 117 \\
\hline LM 2515 D & LM2272P & 115 & 110 \\
\hline LM 2509 D & LM2255P & 115 & 113 \\
\hline LM 3005 D & LM1 594P LM2236P & 114 & 114 \\
\hline LM $2531 \mathrm{D}$ & LM2255P, LM2449T & 117 & 116 \\
\hline \multicolumn{2}{|c|}{ Rappel moyenne de b catégorie } & $(112)$ & (111) \\
\hline \multicolumn{2}{|c|}{$\begin{array}{l}\text { Mbyenne des croisements retenus } \\
\text { (et après régression génophénotypique) }\end{array}$} & $116(115)$ & $114(114)$ \\
\hline \multicolumn{4}{|c|}{ Catégorie hybrid e C2501 } \\
\hline LM 3037 D & LM2256P & 121 & 110 \\
\hline LM 3053 D & LM2250P, LM5909T & 117 & 112 \\
\hline LM 3034 D & LM2453T & 131 & 118 \\
\hline LM 3040 D & LM3944T, PO2973P & 113 & 113 \\
\hline LM 3604 D & LM2255P & 121 & 112 \\
\hline LM 3033 D & LM3391T & 121 & 110 \\
\hline LM 3869 D & LM 2466P & 116 & 112 \\
\hline LM $3621 \mathrm{D}$ & LM1 594P, LM6351T & 115 & 106 \\
\hline LM 3038 D & LM1571 P, LM 2038 T & 118 & 116 \\
\hline \multicolumn{2}{|c|}{ Rappel moyenne de b catégorie } & (113) & $(107)$ \\
\hline \multicolumn{2}{|c|}{$\begin{array}{l}\text { Mbyenne des croisements retenus } \\
\text { (et après régression génophénotypique) }\end{array}$} & $119(118)$ & $112(112)$ \\
\hline
\end{tabular}

Tableau 3. Croisements retenus dans deux catégories hybrides pour la production de semences 


\begin{tabular}{|lcc}
\hline Temps & Test comparatif & Préparation de la sortie variétale \\
\hline An 1 & Plantation du test & \\
An 2 & & \\
An 3 & & \\
An 4 & Entrée en production & Réalisation des autofécondations de tous les pa rents \\
An 5 & & \\
An 6 & Premières interpréta tions & \\
An 7 & & \\
An 8 & & \\
An 9 & & \\
An 10 & Fin de l'essais : résultats & Début de l'entrée en production de semences après cho ix final \\
An 11 & & \\
An 12 & & \\
An 13 & & Pleine explondations retenues après un premier cho ix \\
An 14 & &
\end{tabular}

Tableau 4. Chronologie théorique de l'exploitation d'un test comparatif de croisements

\begin{tabular}{|lrrrcrc}
\hline Région & Sud-Est & Abidjan & $\begin{array}{c}\text { Centre- } \\
\text { Ouest }\end{array}$ & $\begin{array}{c}\text { Sud- } \\
\text { Ouest }\end{array}$ & $\begin{array}{c}\text { Hors } \\
\text { secteur }\end{array}$ & Total \\
\hline \%échantillon & 29,8 & 6,5 & 40,4 & 19,1 & 4,2 & 100 \\
Création & 75,1 & 70,8 & 75,0 & 39,1 & 75,5 & 67,9 \\
Extention & 20,2 & 26,2 & 23,5 & 60,9 & 24,5 & 29,9 \\
Replantation & 4,6 & 3,0 & 1,5 & 0 & 0 & 2,2 \\
\hline
\end{tabular}

Tableau 5. Répartition des surfaces créées ou en extention ou en replantation par région4Échantillon : 715 planteurs individuels reçus à la Mé du 27/12/1999 au 29/04/2000 pour 498831 graines vendues. 